\title{
Infancias Imágenes
}

http://revistas.udistrital.edu.co/ojs/index.php/infancias

DOI: 10.14483/udistrital.jour.infimg.2015.2.a06

IMÁGENES DE INVESTIGACIÓN

\section{Hacer visible el pensamiento: alternativa para una evaluación para el aprendizaje $^{*}$}

\author{
Thinking Made Visible: An Alternative in Assessment for Learning \\ María Yaned Morales Benítez** Ignacio Restrepo Uribe
}

Para citar este artículo: Morales, M. Y.; Restrepo, I. (2015). Hacer visible el pensamiento: alternativa para una evaluación para el aprendizaje. Infancias Imágenes, 14(2), 89-100.

Recibido: 26-julio-2015 / Aprobado: 01-septiembre-2015

\section{Resumen}

Este artículo recoge reflexiones a partir de los resultados de la investigación "La visibilización del pensamiento: una herramienta esencial en la evaluación para el aprendizaje", con base en los conceptos de evaluación para el aprendizaje, evaluación formativa, Enseñanza para la Comprensión y visibilización del pensamiento. La propuesta se desarrolló desde lo cualitativo con un diseño de investigación acción, y con la cual se determinó la necesidad de entender que la evaluación para el aprendizaje debe llevar al estudiante y al docente a visibilizar el pensamiento, a mejorar sus comprensiones, a lograr los objetivos propuestos, a tener en cuenta el punto de vista del estudiante, a generar procesos de retroalimentación y a fortalecer sus aprendizajes.

Palabras clave: evaluación, comprensión, aprendizajes, retroalimentación, pensamiento

\begin{abstract}
This article gathers reflections from the results of the investigation "The visualization of the thought: an essential tool in the evaluation for learning", based on the concepts of assessment for learning, formative assessment, teaching for understanding and visibility of thought. The proposal was developed from and where it was determined the need to understand that assessment for learning must lead both student and teacher to visualize thinking, to improve their understanding, to achieve the proposed objectives, to take into account the point of view of the student, to generate a feedback process, and to strengthen their learning.
\end{abstract}

Keywords: assessment, understanding, learning, feedback, thinking

* Este artículo reflexivo es producto del proyecto de investigación "La visibilización del pensamiento: una herramienta esencial en la evaluación para el aprendizaje", desarrollada entre julio de 2013 y mayo de 2015, para optar al título de magíster en Pedagogía de la Universidad de la Sabana.

** Magíster en pedagogía, Universidad de la Sabana. Especialista en Pedagogía de la Comunicación y Medios Interactivos, Universidad Distrital Francisco José de Caldas. Candidata a magíster en Pedagogía, Universidad de la Sabana. Docente de Español en Secundaria y Media, Colegio Rodolfo Llinás I.E.D. Correo electrónico: mariamobe@unisabana.edu.co

*** Economista Universidad Javeriana. Magíster en Educación, Pontificia Universidad Javeriana. Docente de la Maestría en Pedagogía, Universidad de la Sabana. Coordinador del currículo Gimnasio Moderno. Correo electrónico: ignacio.restrepo@unisabana.edu.co 


\section{UNA MIRADA A LA PROPIA PRÁCTICA}

Este trabajo de investigación nace de la autorreflexión sobre las propias prácticas de evaluación en la asignatura de Español, de grado décimo, dado que allí se evidencia un alto número de estudiantes que reprueban la asignatura en cada periodo escolar, y por la que se percibe una alta desmotivación.

En este sentido se hace necesario establecer una nueva estrategia que permita que los estudiantes encuentren en la evaluación una posibilidad de aprendizaje, de hacer visible su pensamiento y que, a su vez, sientan motivación por la asignatura; esto ayudará a mejorar y enriquecer la práctica pedagógica.

Así, el ejercicio de autoevaluación por parte del docente se podría constituir también en el primer paso de transformación de los procesos de evaluación en el aula; es pertinente, igualmente, que el discurso de evaluación formativa y para el aprendizaje trascienda los anaqueles de los libros y los conversatorios académicos, y se constituya en una realidad que redunde en la calidad de la educación. En consecuencia, la evaluación formativa debe ser el apoyo oportuno y pertinente que aporte al mejoramiento de las prácticas educativas y los procesos de enseñanza/ aprendizaje. El primer paso para lograrlo es mediante la autorreflexión sobre el propio desempeño como docentes, pues reconocer en qué se está fallando o identificar las potencialidades facilitan, desde un ejercicio crítico, mejorar, comprender y transformar la evaluación. Según Álvarez Méndez (2011), "cuando una evaluación aspira a ser formativa tiene que estar al servicio de la práctica para poder mejorar y sobre todo estar al servicio de quienes participan en ella" (p. 15). Así que la revisión de las propias prácticas como docentes, al tener en cuenta el sentir de los estudiantes, se puede asumir como parte de la evaluación formativa y para el aprendizaje.

\section{UNA EVALUACIÓN FORTALECEDORA DE PROCESOS DE ENSEÑANZAY APRENDIZAJE}

Dentro de la dinámica escolar, a la evaluación tradicionalmente se la ha atribuido una función sancionatoria, lo que hace que sus propósitos reales de potencializar los procesos de enseñanza/aprendizaje, mejorar las prácticas, generar retroalimentación, optimizar las compresiones y lograr reflexiones en torno a las fortalezas y debilidades, se pierdan. El hecho de ser una evaluación sancionatoria implicaría pensar en una evaluación sumativa para certificar si los aprendizajes están bien o mal. Desde esta mirada, la nota, aunque importante, no puede constituirse en el eje central del proceso evaluativo, ya que genera ansiedad y temor. La evaluación pensada con un fin formativo y para el aprendizaje utiliza la información para lograr cumplir los objetivos propuestos. Para Malbergier y Campelo (2009), "cuando no se enfoca la evaluación en la calificación se puede hacer uso de la información que se obtiene para adaptar la enseñanza de modo tal que les permita avanzar hacia el logro de sus objetivos" (p. 25).

Así pues, la evaluación formativa cumple la función de regular las actividades de enseñanza/ aprendizaje (Feldman, 2010). Este tipo de evaluación puede ser efectiva si se adecua a la individualidad en el proceso de aprendizaje. Por tanto, la evaluación para el aprendizaje debe ser una herramienta que potencialice los aprendizajes, fortalezca los conocimientos adquiridos y dé cuenta de las nuevas comprensiones por parte de los estudiantes. "Solo cuando aseguramos el aprendizaje, podremos asegurar la evaluación. La buena evaluación que forma, convertida ella misma en medio de aprendizaje y en expresión de saberes. Solo entonces podemos hablar de evaluación formativa" (Álvarez Méndez, 2011, p. 12).

Un concepto recurrente dentro de lo que se ha expresado hasta ahora es el de aprendizaje, y la evaluación no se puede desligar de él; la evaluación para el aprendizaje, como lo argumenta López (2013), "es un medio por el cual se documentan los aprendizajes, logrando así que se alcancen los objetivos que se han planteado" (p. 21). Es la posibilidad, si la evaluación está bien planteada, de que los estudiantes hagan visible su pensamiento y así mismo puedan comprender mejor esos nuevos aprendizajes y asociarlos con conocimientos previos. 
Para que la evaluación formativa y para el aprendizaje sea posible, debe cumplir cinco condiciones (López, 2013, p. 23), que se detallan en el gráfico 1.

Gráfico 1. Condiciones para la evaluación

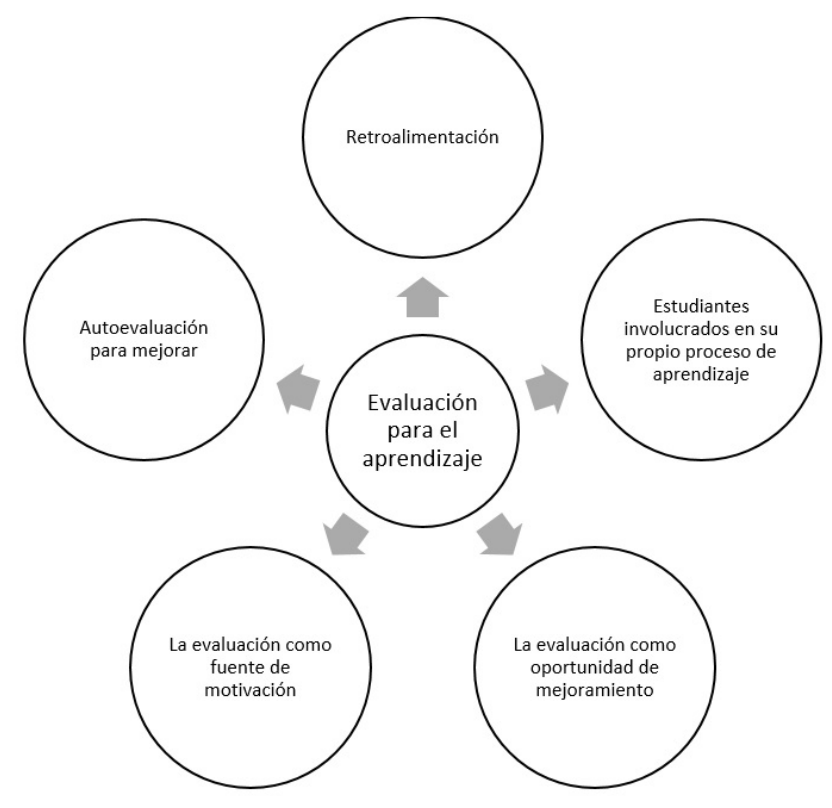

Fuente: elaboración propia (agosto de 2014)

Estas condiciones, tal como las plantea el autor, denotan una fuerte necesidad de incluir a los estudiantes en sus procesos de formación, de generar una cultura de la evaluación más funcional y dinámica; una evaluación que permita desarrollar la metacognición, con lo cual el maestro enriquece sus prácticas, y el estudiante mejora sus procesos.

Dentro de las cinco condiciones que menciona el autor hay dos que son particularmente importantes:

a. Retroalimentación, como posibilidad de que el estudiante sepa en donde está en su propio proceso (López, 2013).

b. Autoevaluación y coevaluación como la forma en que los estudiantes asuman la responsabilidad de monitorear su aprendizaje.

La retroalimentación es fundamental para que los estudiantes tengan claro dónde se ubican en su proceso; se constituye en una excelente alternativa de aprendizaje ya que así se fortalecen los aspectos positivos y se reconocen en situaciones difíciles una oportunidad de mejoramiento. Esto solo se logra mediante un pensamiento en el cual el estudiante pueda analizar su aprendizaje y establecer, junto al maestro, nuevos caminos a seguir. De este modo, la retroalimentación será realmente eficaz.

Por su parte, es muy común dentro de la escuela que la evaluación en el aula sea hecha solo por el maestro; es decir, que prime la heteroevaluación, mientras que en los espacios en los que la autoevaluación y la coevaluación tienen presencia no se les da la importancia que estas tienen. Pareciera ser que la opinión del estudiante no es tan relevante y que él forme parte de su propio proceso no se considera necesario. La autoevaluación y la coevaluación tienen unas funciones y características que las hacen necesarias durante el aprendizaje y que ayudan a que el alumno visibilice su pensamiento, su sentir frente a su quehacer académico.

"La autoevaluación es la oportunidad que tienen los estudiantes de participar activamente en un proceso reflexivo y analítico de sus aprendizajes y fomenta el aprendizaje autónomo" (López, 2013, p. 79); además es una fuente de motivación para el estudiante, quien hace conciencia de fortalezas y debilidades para mejorar, su opinión es tenida en cuenta y su trabajo valorado en su dimensión real.

En segundo lugar, la coevaluación facilita que los estudiantes evalúen los desempeños de sus compañeros; esto beneficia también al que evalúa, pues pone a prueba sus conocimientos, Ilevándolo a hacer asociaciones. Por otra parte, es importante porque hace visible su pensamiento a través de su capacidad de análisis y su criterio para evaluar a su compañero; el alumno evaluado, al tener la mirada de un par académico, se sentirá en igualdad de condiciones, será una oportunidad para aprender del otro e intercambiar ideas.

También se puede pensar, y aunque no es tema de esta investigación, en una forma de aprendizaje cooperativo, puesto que hay un apoyo mutuo en la coevaluación, se respalda el trabajo del 
compañero, se hace un ejercicio responsable de retroalimentación y se construyen nuevos saberes.

Los estudiantes que trabajan (aprenden juntos) se implican más activamente en el proceso de aprendizaje, puesto que las técnicas de aprendizaje cooperativo permiten a los estudiantes actuar sobre su propio proceso de aprendizaje, implicándose más con la materia de estudio y con sus compañeros. Capitaliza la capacidad que tienen los grupos para incrementar el nivel de aprendizaje mediante la interacción con sus compañeros. (Domingo, 2008, p. 232).

En este sentido se pueden generar más allá de la coevaluación grupos de estudio donde la retroalimentación individual y colectiva enriquezca los aprendizajes.

En síntesis, uno de los propósitos de la evaluación para el aprendizaje es proporcionar información sobre los aprendizajes de los estudiantes y, a partir de allí, determinar las acciones a seguir (Álvarez Méndez, 2011) .Y aun se puede Ilegar más lejos cuando va acompañada por la visibilización del pensamiento, porque no solamente se trata de recolectar información de aprendizaje, consiste en que esa información recoja de manera efectiva las comprensiones de los estudiantes; que sea una valoración continua, de doble vía: el estudiante aprende, mejora, transforma, comprende y dinamiza, y el maestro, a su vez, con todo lo que sus estudiantes aportan, hace lo propio.

Pero para lograr todo esto, es imperativo responder de manera objetiva, crítica y con un propósito de transformación en el aula las siguientes preguntas: ¿Qué tanto se está enseñando? ¿Para qué sirve? ¿Realmente aprendieron los alumnos? ¿Se han evidenciado sus comprensiones? ¿Hay una visibilización real del pensamiento? El primer paso, sin dudas, es una revisión juiciosa de la propia práctica que permita evidenciar las falencias para transformarlas y potencializar las fortalezas, logrando así una mejor dinámica en el proceso de enseñanza/aprendizaje.

\section{EL PENSAMIENTO DE LOS ESTUDIAN- TES: UN MUNDO DE OPORTUNIDADES}

Hacer visible el pensamiento es una tarea fundamental dentro de la escuela, solo así se podrán tener en cuenta las comprensiones previas y posteriores de los estudiantes. Sin embargo, no es algo de lo cual se tenga conciencia en la práctica, quizá porque se supone que se está haciendo. "La gente es indiferente ante situaciones que invitan a pensar" (Perkins, 2003). Normalmente tiende a confundirse el ejercicio de pensamiento con la memorización; esta última es importante en ciertos casos, pero se debe entender que memorizar muchos conceptos no implica necesariamente lograr comprensiones.

Por consiguiente, el proceso de pensamiento implica un ejercicio juicioso que conduzca a la construcción de nuevos saberes, de enlazarlos con saberes previos; fundamentalmente implica el compromiso del docente por hacer que su clase sea un espacio propicio para que esto sea una realidad en el aula. Con la siguiente metáfora se puede evidenciar mejor su importancia: "Cuando hacemos visible el pensamiento no obtenemos solo una ventana a lo que entienden los estudiantes, sino también la forma en que estamos entendiéndolos" (Ritchhart, Church y Morrison, 2011, p. 27).

La posibilidad de hacer visible el pensamiento y que los estudiantes lo hagan de forma permanente, debe orientar de manera efectiva en la evaluación, puesto que pensar ayuda a comprender mejor los conceptos y, sobre todo, a aplicarlos en contextos reales. Por su parte, la evaluación, si está bien fundamentada, aporta a la visibilización del pensamiento, ya que como estrategia de aprendizaje, es una herramienta fundamental en el aula. "En busca de una cultura de pensamiento, la noción de hacer visible el pensamiento ayuda a concretar lo que debe ser en el aula y ofrece la orientación para hacerlo" (Perkins, 2003).

Adicionalmente, hacer visible el pensamiento contribuye a tener procesos eficaces de aprendizaje, pues el estudiante tiene la oportunidad de 
expresar sus ideas, conocimientos previos, sentimientos sobre lo que hace. Tal como lo dicen Ritchhart, Church y Morrison (2011, p. 27): que ese conocimiento sirva para apoyar y participar en el proceso de conocimiento .

Generar una cultura de pensamiento en la escuela es fundamental porque así es posible encontrar el deber ser en el aula (Perkins, 2003). Visibilizar el pensamiento de los estudiantes los hace más conscientes de sus aprendizajes, los conduce a reconocer sus potencialidades y debilidades, y los hace partícipes de su propio aprendizaje.

Por otra parte, la visibilización del pensamiento se constituye en una herramienta al momento de evaluar, ya que al evidenciar las comprensiones de los estudiantes se tendrá claridad sobre sus avances, y a través de la retroalimentación se podrá reforzar aquello que ellos necesitan.

\section{AL RITMO DEL PENSAMIENTO}

Para lograr que los estudiantes visibilicen su pensamiento existen una serie de actividades, las cuales se denominan rutinas de pensamiento ${ }^{1}$, y mediante estrategias lúdicas, dinámicas y entretenidas acercan a los estudiantes y al docente a su propio conocimiento. Ritchhart, Church y Morrison (2011) las definen como herramientas que promueven el pensamiento; estas, al ser tan dinámicas, no solo fomentan el pensamiento sino también motivan al estudiante en el proceso de aprendizaje (p. 29).

De acuerdo con su intencionalidad, las rutinas de pensamiento se clasifican en tres grupos (gráfico 2).

Sin embargo estas rutinas requieren de la disposición del docente para incluirlas en el desarrollo de la clase, puesto que implican preparación, retroalimentación de las respuestas, organización del

Gráfico 2. Algunas rutinas de pensamiento

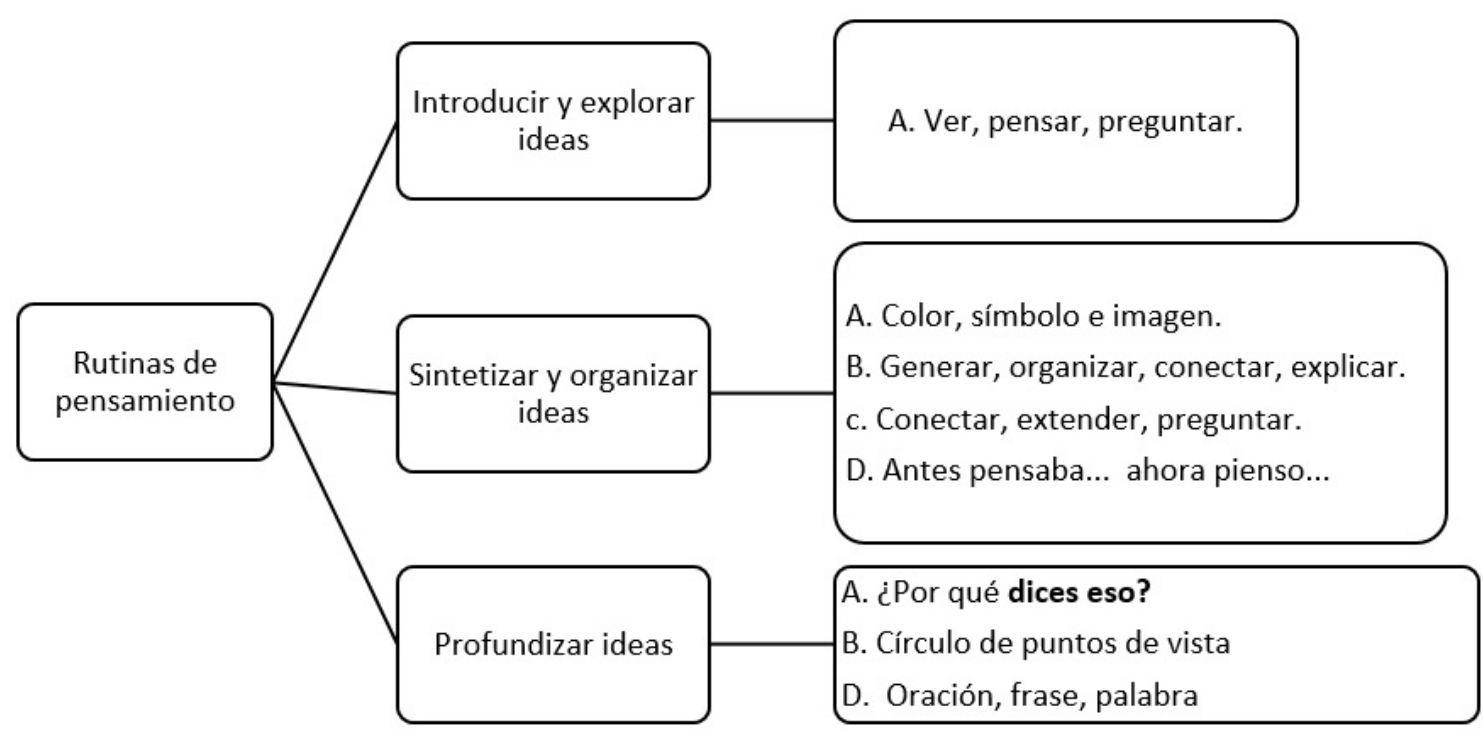

Fuente: elaboración propia (agosto de 2014)

1. Las rutinas de pensamiento son herramientas que permiten visibilizar el pensamiento. Tienen ventajas como ser dinámicas, trabajarse de forma individual o grupal, permiten las interrelaciones entre docente/estudiante, estudiantes y conocimiento, estudiantes con sus pares académicos; además facilitan la discusión y son adaptables a las necesidades propias de cada grado o estudiante.

Perkins (2003) las define así: "[...] las rutinas de pensamiento son patrones sencillos de pensamiento que pueden ser utilizados una y otra vez, hasta convertirse en parte del aprendizaje de la asignatura misma". 
curso y mente abierta para escuchar los múltiples pensamientos, encausarlos, corregirlos, apoyarse en ellos; es decir, hacer del aula de clase un lugar donde los estudiantes puedan hacer visible su pensamiento de una manera diferente y cautivadora. Ritchhart, Church y Morrison (2011) plantean:

Hacer visible el pensamiento no está exento de desafíos. Como hemos discutido, primero tenemos que tener claro en nuestra mente lo que es el pensamiento. Esto nos permite hacer el pensamiento visible al nombrarlo y darse cuenta en qué medida se produce. Además para que pensar ocurra en los estudiantes deben primero tener algo en qué pensar (p. 30).

De otra parte, las rutinas de pensamiento hacen que la comprensión no sea cuestión de memorización, pues no es lo mismo repetir una serie de conceptos que llevarlos a un contexto que se puede explicar. Dentro de la investigación realizada se evidenció una situación que muestra la eficacia de esta estrategia: es el caso de una estudiante con déficit cognitivo leve, que no tiene el mismo ritmo de aprendizaje de sus demás compañeros. A pesar de esta limitación, ella respondió muy bien a todas las actividades planteadas en la asignatura, obviamente con las limitaciones de su condición; sus avances en el transcurso del curso han sido positivos, la visibilización del pensamiento ha hecho que ella explore su pensamiento y pueda plasmar sus nuevas comprensiones. Español fue la única asignatura que no hizo flexibilización curricular puesto que la estudiante ha demostrado que con la estrategia implementada puede responder con la materia. Es importante aclarar que hubo momentos en los cuales presentó dificultades, pero con la retroalimentación las superó.

Es claro que las limitaciones las ponemos nosotros mismos, y es importante entender que todo estudiante tiene algo que decir, que compartir, unos conocimientos previos que se hace necesario explorar e incluir en las dinámicas evaluativas de clase. Por último, las rutinas de pensamiento facilitaron un proceso de evaluación efectiva, pues logró visibilizar el pensamiento de los estudiantes de una forma diferente, como lo afirma Ritchhart, Church y Morrison (2011), más allá de la memorización, trabajos y actividades. Una evaluación que tuvo en cuenta los saberes de los estudiantes, sus intereses e inquietudes.

\section{UNIDADES DE CLASE QUE INVITAN A LA COMPRENSIÓN: UNA NUEVA FOR- MA DE PENSAR}

Hablar en términos de comprensión parecería un discurso trillado, pero ante este concepto surgen varias preguntas que se dan en el marco de la práctica misma. Cuando planeamos las clases es pertinente reflexionar sobre lo que se pretende con ellas; para ello resulta de gran ayuda responder a los siguientes interrogantes: ¿Tenemos un objetivo claro? ¿Qué esperamos al finalizar el proceso de nuestros estudiantes, que tengan una buena nota o que realmente hayan comprendido? ¿Tenemos estrategias claras y definidas para saber si los estudiantes realmente comprendieron? ¿La evaluación está pensada en términos de lo que comprendieron y no de lo que memorizaron? Estas preguntas fueron las que dieron origen a la idea de pensar en un proyecto que mirara la evaluación en términos de comprensión y no de memorización, entendiendo que la Enseñanza para la Comprensión (EpC) no rechaza la memorización, pues en muchas ocasiones antecede al aprendizaje.

Estas deben ser las preguntas que orienten la preparación de cada tema de clase, con formas dinámicas en el proceso de enseñanza, donde las motivaciones de los estudiantes frente al conocimiento aumenten, puesto que sus saberes son tenidos en cuenta, donde la variedad en el desarrollo de los desempeños se dinamice y la valoración, al ser continua, tenga en cuenta y valore todo el proceso, y le ayude a mejorar de forma permanente. 
Pensar en unidades bajo el modelo de la $\mathrm{EpC}^{2}$ ha facilitado visibilizar mucho más el pensamiento de los estudiantes, por el diseño con el cual están planteadas. Stone (1999) señala que comprender no es memorizar, sino es un proceso en el cual los estudiantes establecen relaciones entre los conceptos previos y los nuevos.

Antes de hablar de EpC se debe determinar qué es la comprensión. De acuerdo con Perkins (1999), "comprender es la habilidad de pensar y actuar con flexibilidad a partir de lo que uno sabe. Es la capacidad del desempeño flexible" (p. 69). Comprender entonces no es memorizar, es establecer relaciones entre los conceptos previos, los que ya traen los estudiantes y los nuevos, y cómo eso que se ha comprendido se puede llevar a la práctica misma de formas dinámicas. Stone (1999) habla de formas novedosas.

Sin embargo, para entender la EpC es importante tener en cuenta sus principios generales que Perkins (como se citó en Martínez, 2007) presenta así:

- El aprendizaje para la comprensión se produce principalmente por medio de un compromiso reflexivo con desempeños de comprensión posibles de abordar pero que se presentan como un desafío.

- Los nuevos desempeños de comprensión se construyen a partir de comprensiones previas y de la nueva información ofrecida por el entorno institucional.

- Aprender un conjunto de conocimientos y habilidades para la comprensión infaliblemente exige una cadena de desempeños de comprensión de variedad y complejidad crecientes.
- El aprendizaje para la comprensión a menudo implica un conflicto con repertorios más viejos de desempeños de comprensión y con sus ideas e imágenes asociadas.

Además de estos principios, la EpC tiene un marco conceptual que lo sustenta y que se puede considerar como la carta de navegación para el desarrollo mismo de la práctica en el aula, a la luz de este modelo. El marco conceptual está constituido por cuatro elementos: tópicos generativos, metas de comprensión, desempeños de comprensión y valoración continua ${ }^{3}$.

En la tabla 1 se presenta un modelo de unidad de clase para grado décimo en clase de Español. Con el desarrollo de la unidad con los parámetros establecidos por la EpC se lograron resultados que fueron más allá de lo esperado: no solo se logró que las comprensiones de los estudiantes fueran satisfactorias y conocer sus pensamientos acerca de la temática planteada, sino que además se logró que se generaran procesos de discusión, debate argumentado, trabajo en equipo.

Teniendo en cuenta las características propias de la EpC se puede plantear una evaluación integral y formativa en la que el estudiante participe en su propio proceso de aprendizaje y realmente llegue a la comprensión. La evaluación es entonces una herramienta que facilita la retroalimentación de cada fase del desarrollo mismo del aprendizaje. Por su parte, al maestro le ayuda a dinamizar las prácticas en el aula y a enriquecer sus propios conocimientos con aquellos que traen los estudiantes, así como conocer el contexto en el que desarrolla su labor docente.

2. Enseñanza para la Comprensión: estrategia propuesta por el Proyecto Zero de la Escuela de Educación de Harvard, que busca fomentar las comprensiones de los estudiantes desde una perspectiva constructivista por desempeños.

3. Tópicos generativos: son los grandes temas que se abordan en clase y que deben responder a las siguientes características: centrales para un dominio o disciplina; accesibles e interesantes para los alumnos; interesantes para el docente; rico en conexiones. Metas de comprensión: es lo que se espera que los alumnos lleguen a comprender. Definen los procesos, preguntas o relaciones que los estudiantes comprenderán mejor por medio de su indagación. Dispuestas en una estructura compleja. Explicitas y publicas Centrales para una materia.

Desempeños de comprensión: es el elemento más importante dentro de este marco conceptual. Las actividades son desempeños de comprensión solo si desarrollan y demuestran claramente la comprensión por parte de los alumnos de metas de comprensión importantes. Los estudiantes aplican sus conocimientos previos y los nuevos de forma dinámica. Evaluación diagnostica continua: la evaluación diagnóstica se hace durante todo el proceso. Tiene en cuenta: criterios relevantes, explícitos y públicos. Evaluaciones diagnosticas continuas. Múltiples fuentes. Estimar el avance y configurar la planificación" (Stone, 1999). 
Tabla 1. Unidad EpC "Libertad de expresión en Colombia"

\begin{tabular}{|c|c|c|c|c|c|}
\hline \multicolumn{6}{|c|}{ Unidad 2 (Libertad de expresión en Colombia) } \\
\hline \multicolumn{6}{|c|}{ Tópico generador: Exprésate con libertad } \\
\hline \multicolumn{6}{|c|}{ Metas de comprensión } \\
\hline \multicolumn{2}{|c|}{$\begin{array}{l}\text { 1. Los estudiantes } \\
\text { comprenderán los } \\
\text { diferentes aspectos } \\
\text { de la libertad de } \\
\text { expresión. } \\
\text { Pregunta: } \\
\text { ¿Cuáles son los } \\
\text { aspectos principales } \\
\text { de la libertad de } \\
\text { expresión? }\end{array}$} & $\begin{array}{c}\text { 2. Los estudiantes } \\
\text { comprenderán las } \\
\text { diferentes situaciones } \\
\text { en las que se } \\
\text { vulnera la libertad } \\
\text { de expresión y lo } \\
\text { expresarán en un } \\
\text { debate } \\
\text { Pregunta: } \\
\text { ¿En qué situaciones } \\
\text { específicas se } \\
\text { vulnera la libertad de } \\
\text { expresión? }\end{array}$ & \multicolumn{2}{|c|}{$\begin{array}{l}\text { 3. Los estudiantes } \\
\text { comprenderán } \\
\text { la importancia } \\
\text { de expresar las } \\
\text { ideas de forma } \\
\text { argumentada. } \\
\text { Pregunta: } \\
\text { ¿Por qué es } \\
\text { importante la } \\
\text { argumentación de } \\
\text { las ideas? }\end{array}$} & $\begin{array}{l}\text { 4. Los estudiantes } \\
\text { comprenderán la } \\
\text { importancia de } \\
\text { expresar el punto } \\
\text { de vista y respetar el } \\
\text { punto de vista de los } \\
\text { demás. } \\
\text { Pregunta: } \\
\text { ¿Cómo generar } \\
\text { acciones de respeto } \\
\text { frente a las opiniones } \\
\text { de los demás? }\end{array}$ \\
\hline MC & \multicolumn{2}{|c|}{ DESEMPEÑOS DE COMPRENSIÓN } & TD & \multicolumn{2}{|c|}{ VALORACION CONTINUA } \\
\hline 1 y 2 & \multicolumn{2}{|c|}{$\begin{array}{l}\text { Exposición oral de sus opiniones en } \\
\text { torno a las siguientes preguntas: } \\
\text { ¿En qué momentos sientes que no } \\
\text { puedes expresar tus ideas libremente } \\
\text { y por qué? ¿Respetas las opiniones de } \\
\text { los demás? ¿Cómo sabes que lo haces? } \\
\text { O, por el contrario, no has respetado la } \\
\text { opinión de los demás ¿Qué has hecho? } \\
\text { Elaboración de un cuento que sintetiza } \\
\text { sus respuestas orales }\end{array}$} & $\mathrm{E}$ & \multirow{3}{*}{\multicolumn{2}{|c|}{$\begin{array}{l}\text { Cada actividad tendrá una } \\
\text { retroalimentación, de parte del } \\
\text { docente y de sus compañeros. } \\
\text { Por otra parte se diseñaron } \\
\text { dos matrices de evaluación y } \\
\text { autoevaluación para que los } \\
\text { estudiantes hagan un análisis de su } \\
\text { propio proceso. } \\
\text { Al comenzar la unidad los } \\
\text { estudiantes tienen claro cuáles son } \\
\text { los objetivos que se buscan y a dónde } \\
\text { se quiere llegar y cuáles son los pasos } \\
\text { para lograrlo. }\end{array}$}} \\
\hline 1 & \multicolumn{2}{|c|}{$\begin{array}{l}\text { Los estudiantes elaboran una oración, } \\
\text { frase y palabra para sintetizar sus } \\
\text { aprendizajes }\end{array}$} & IG & & \\
\hline 3 y 4 & \multicolumn{2}{|c|}{$\begin{array}{l}\text { Elaboración de un escrito como } \\
\text { respuesta a: ¿Cómo ven los jóvenes } \\
\text { de hoy la libertad de expresión en } \\
\text { Colombia y qué aportes pueden hacer a } \\
\text { este tema? }\end{array}$} & PF & & \\
\hline oye & $\begin{array}{l}\text { nes: MC: en } \\
\text { e lugar se es } \\
\text { nal de síntesi }\end{array}$ & $\begin{array}{l}\text { lugar se escribe el núr } \\
\text { e el tipo de desempe }\end{array}$ & de & $\begin{array}{l}\text { eta a la c } \\
\text { oración. I }\end{array}$ & $\begin{array}{l}\text { dirige este desempeño. } \\
\text { vestigación guiada. PF: }\end{array}$ \\
\hline
\end{tabular}

Fuente: elaboración propia (marzo de 2014) 
Es claro que pensar en unidades de comprensión no es sencillo, requiere de tiempo y de una conciencia plena de lo que se busca con cada una de ellas. Sin embargo, constituye una excelente herramienta de visibilización del pensamiento que brinda la posibilidad de una evaluación permanente que incluya el sentir de los estudiantes, haciéndolos partícipes de su propia evaluación y generando un grado de confianza con sus compañeros y docentes; también fortalece las comprensiones de las temáticas con cada retroalimentación y hace sentir a los estudiantes parte del desarrollo de la clase. "Solo si el estudiante participa activa y libremente en esta evaluación podrá sentirla como una acción de apoyo y estímulo que lo involucra y compromete, y no como un juicio extremo que califica o descalifica sus logros" (Jaramillo, Escobedo y Bermúdez, 2004, p. 533).

\section{MATRICES DE EVALUACIÓN: UNA RUTA A SEGUIR PARA EL MEJORAMIENTO}

Las matrices de evaluación se pueden considerar como una herramienta que dan paso a la visibilización del pensamiento de los estudiantes, pues ellos deben reflexionar sobre su propio desempeño, el del docente y el de sus compañeros; cumpliendo así con uno de los objetivos de la autoevaluación y que es extensible a la co- y a la heteroevaluación. "La reflexión es un componente esencial de la autoevaluación. Es un proceso que supone un pensamiento orientado hacia las acciones pasadas, que se lleva a cabo para mejorar y obtener enseñanzas con relación al futuro" (Malbergier y Campelo, 2009, p. 29).

Es importante tener en cuenta que las matrices han sido construidas a partir de los intereses de los estudiantes ${ }^{4}$; esto ha ayudado a que ellos asuman con mayor seriedad y compromiso el trabajo con esta herramienta.

Otro aspecto importante para que los estudiantes recurran a estas matrices con mayor agrado es el lenguaje utilizado en ellas; un lenguaje sencillo, cercano y alejado de lo punitivo; que busca que el alumno entienda en qué parte del proceso está, sin cuestionamientos sino haciéndole sentir que su trabajo es valorado. En la tabla 2 se puede observar un modelo de matriz de autoevaluación.

Según Freire (citado por Mesa, 2009), "el proceso educativo tiene que fundamentarse en la participación, la autorreflexión y el pensamiento crítico de quienes son sujetos del proceso de enseñanza y de aprendizaje, esto es, las y los estudiantes y las y los docentes" (p. 3). Las matrices se constituyen en una gran fuente de autorreflexión, pues conducen, mediante unos parámetros claros, a que los estudiantes sean objetivos, concisos, y a partir de allí, transformen o consoliden los desempeños académicos y convivenciales.

\section{MÁS ALLÁ DE LA INVESTIGACIÓN: AL- GUNAS RECOMENDACIONES FINALES}

Una investigación no termina con la entrega del informe escrito ya que surgen nuevas inquietudes, necesidades y expectativas para que las conclusiones y propuestas que se dieron se hagan visibles para todos y cumplan con su objetivo de contribuir a la transformación de las prácticas educativas; por ello, y a manera de propuesta, se hacen las siguientes recomendaciones:

- Hacer un proceso de socialización de la propuesta con docentes de otras instituciones educativas; compartir la visión de la visibilización del pensamiento, las rutinas de pensamiento y la evaluación para el aprendizaje, de tal manera que transcienda el espacio donde se desarolló el proyecto.

- Dar continuidad al proceso, no solo con un grupo focal, sino con todos los estudiantes para visibilizar aún más los alcances que esta propuesta ha tenido y, en consecuencia, continuar con el mismo entusiasmo, dado que los

4. A través de encuesta sobre percepciones de evaluación y encuesta sobre aspectos que deben ser tenidos en cuenta al momento de hacer una auto-, hetero- y coevaluación en el ámbito convivencial y académico. 
Tabla 2. Matriz de autoevaluación, unidad de comprensión

\begin{tabular}{|c|c|c|c|c|}
\hline $\begin{array}{l}\text { ASPECTO A } \\
\text { EVALUAR }\end{array}$ & AÚN NOS FALTA & PODEMOS MEJORAR & $\begin{array}{l}\text { POR BUEN } \\
\text { CAMINO }\end{array}$ & $\begin{array}{c}\text { LLEGAMOS A LA } \\
\text { META }\end{array}$ \\
\hline $\begin{array}{c}\text { Nivel de } \\
\text { comprensión } \\
\text { de la temática }\end{array}$ & $\begin{array}{l}\text { Presento dificultades } \\
\text { en la comprensión de } \\
\text { algunos temas, incluso } \\
\text { al punto de no poder } \\
\text { identificar los aspectos } \\
\text { de dichos temas. }\end{array}$ & $\begin{array}{l}\text { Presenté algunas di- } \\
\text { ficultades en la com- } \\
\text { prensión de los temas, } \\
\text { pero logro identificar } \\
\text { algunos aspectos de } \\
\text { dichos temas. }\end{array}$ & $\begin{array}{l}\text { Comprendo } \\
\text { algunos de los } \\
\text { temas dados. }\end{array}$ & $\begin{array}{l}\text { He comprendido todos } \\
\text { los temas planteados } \\
\text { y elaboro ejercicios } \\
\text { para mostrar dicha } \\
\text { comprensión. }\end{array}$ \\
\hline $\begin{array}{l}\text { Disposición } \\
\text { frente a las } \\
\text { actividades } \\
\text { planteadas }\end{array}$ & $\begin{array}{c}\text { No demuestro } \\
\text { ningún interés por el } \\
\text { desarrollo de la clase. }\end{array}$ & $\begin{array}{c}\text { Muestro poco interés } \\
\text { por la actividad } \\
\text { desarrollada o me } \\
\text { distraje con facilidad. }\end{array}$ & $\begin{array}{l}\text { Realizo la activi- } \\
\text { dad, cumplo es- } \\
\text { trictamente con } \\
\text { lo requerido. }\end{array}$ & $\begin{array}{l}\text { Asumo una actitud } \\
\text { de interés, esmero y } \\
\text { disposición frente al } \\
\text { trabajo desarrollado. }\end{array}$ \\
\hline $\begin{array}{l}\text { Trabajo en } \\
\text { equipo }\end{array}$ & $\begin{array}{l}\text { No participo de forma } \\
\text { activa en los trabajos } \\
\text { en grupo. }\end{array}$ & $\begin{array}{l}\text { Mi participación en } \\
\text { el trabajo de grupo es } \\
\text { escasa. }\end{array}$ & $\begin{array}{l}\text { Participo en el } \\
\text { trabajo en grupo } \\
\text { y facilité sus } \\
\text { avances }\end{array}$ & $\begin{array}{l}\text { Asumo una actitud } \\
\text { comprometida y } \\
\text { propositiva con mi } \\
\text { grupo de trabajo. }\end{array}$ \\
\hline $\begin{array}{l}\text { Los aportes } \\
\text { realizados en el } \\
\text { desarrollo de la } \\
\text { unidad }\end{array}$ & $\begin{array}{l}\text { No realizo ningún } \\
\text { aporte al desarrollo de } \\
\text { la unidad. }\end{array}$ & $\begin{array}{l}\text { Los aportes realizados } \\
\text { son muy escasos. }\end{array}$ & $\begin{array}{c}\text { Realizo } \\
\text { algunos aportes } \\
\text { interesantes a la } \\
\text { actividad. }\end{array}$ & $\begin{array}{l}\text { Participo activamente } \\
\text { con aportes interesan- } \\
\text { tes y que enriquecen el } \\
\text { trabajo de todos. }\end{array}$ \\
\hline $\begin{array}{c}\text { Escucha las } \\
\text { participaciones } \\
\text { de los } \\
\text { compañeros }\end{array}$ & $\begin{array}{l}\text { No presto atención a } \\
\text { las intervenciones de } \\
\text { mis compañeros. }\end{array}$ & $\begin{array}{l}\text { La atención a mis } \\
\text { compañeros es escasa } \\
\text { y/o en ocasiones } \\
\text { interrumpo sus } \\
\text { intervenciones. }\end{array}$ & $\begin{array}{l}\text { Escucho a mis } \\
\text { compañeros en } \\
\text { silencio }\end{array}$ & $\begin{array}{c}\text { Escucho atentamente } \\
\text { a mis compañeros y } \\
\text { brindo mis aportes y } \\
\text { opiniones, de manera } \\
\text { respetuosa. }\end{array}$ \\
\hline
\end{tabular}

Fuente: elaboración propia (septiembre de 2014)

estudiantes han notado los cambios y se han beneficiado con ellos.

- Generar una cultura de autoevaluación y coevaluación en los docentes, quienes deben perder el miedo a cuestionarse sobre sus propias prácticas y considerar la visión de los estudiantes; quién mejor que ellos para ayudar al maestro a ver sus fortalezas y debilidades.

- Brindar una posición relevante a los estudiantes en el aula, ya que esto genera motivaciones importantes en ellos y elimina miedos frente a la evaluación.

- Darles voz a los estudiantes en los procesos de evaluación y, también, de construcción de las dinámicas de clase. Ellos tienen grandes aportes por hacer. La construcción de los conocimientos y el ejercicio de comprensión es de doble vía. Ellos aprenden de sus maestros y sus maestros nutren sus clases con los saberes previos que cada uno de los estudiantes trae.

Por último, durante este proceso se generaron algunas preguntas que pueden ser pertinentes para futuras investigaciones:

- ¿Cómo pueden las rutinas de pensamiento constituirse en una alternativa para los estudiantes con déficit cognitivo? 
- ¿Cómo puede la visibilización del pensamiento potencializar la oralidad en los estudiantes?

VISIBILIZANDO EL PENSAMIENTO: REFLEXIÓN PEDAGÓGICA A PARTIR DE LA PROPIA PRÁCTICA DESDE UN ANTES Y UN DESPUÉS

Como conclusión, y siendo consecuentes con el tema de esta investigación, esta reflexión se hará mediante la rutina de pensamiento "Antes pensaba que... Ahora pienso que..." ${ }^{5}$. Es la forma de hacer visible el pensamiento de los autores sobre lo enriquecedor y transformador que ha sido esta investigación. En la tabla 3 se presenta la rutina en dos columnas con las reflexiones que surgen de este proceso investigativo y que llevan a entender que es necesario la transformación de las prácticas evaluativas y la incorporación de escenarios de pensamiento que fomenten, de manera eficaz, las comprensiones de los estudiantes.

Tabla 3. Reflexión pedagógica con la rutina "Antes pensaba que... Ahora pienso que..."

\begin{tabular}{|l|l|}
\hline \multicolumn{1}{|c|}{ Antes pensaba que... } & Ahora pienso que... \\
\hline Evaluar era igual a calificar. & $\begin{array}{l}\text { Evaluar es un proceso continuo y permanente que tiene en cuenta al } \\
\text { estudiante en su integralidad. }\end{array}$ \\
\hline $\begin{array}{l}\text { Los estudiantes no son objetivos para eva- } \\
\text { luar al docente. }\end{array}$ & $\begin{array}{l}\text { Es una práctica que enriquece mi labor como docente, que me per- } \\
\text { mite descubrir los intereses, miedos de mis estudiantes y hace que } \\
\text { yo transformara de forma importante mi práctica educativa. }\end{array}$ \\
\hline $\begin{array}{l}\text { Los estudiantes no tienen los conocimien- } \\
\text { tos suficientes como para incluirlos en el } \\
\text { proceso de construcción de la clase. }\end{array}$ & $\begin{array}{l}\text { No solo tienen las capacidades, sino que además son críticos que ar- } \\
\text { gumentan sus respuestas, que aportan ideas nuevas, que al incluirlos } \\
\text { en los procesos generan un lazo de confianza hacia mí que hace que } \\
\text { tomen gusto por la asignatura. }\end{array}$ \\
\hline $\begin{array}{l}\text { Un estudiante con dificultades de apren- } \\
\text { dizaje genera miedos en el docente por } \\
\text { no saber cómo abordarlo, porque no es } \\
\text { capaz de dar lo que los otros dan. }\end{array}$ & $\begin{array}{l}\text { Las rutinas de pensamiento me han permitido explorar en los cono- } \\
\text { cimientos de todos, sin importar sus limitaciones; esto ha hecho que } \\
\text { sienta una gran satisfacción personal porque he visto sus progresos y } \\
\text { hasta el mejoramiento de su autoestima. }\end{array}$ \\
\hline Memoria igual a comprensión. & $\begin{array}{l}\text { Comprender es mostrar que todos esos conocimientos adquiridos se } \\
\text { aplican de manera adecuada. La construcción de unidades con los } \\
\text { parámetros de la EpC ha facilitado mi práctica; aunque demande } \\
\text { más trabajo en su construcción, la satisfacción de ver cómo los estu- } \\
\text { diantes la aprovechan es muy significativa. }\end{array}$ \\
\hline $\begin{array}{l}\text { Los procesos de investigación solo se ha- } \\
\text { cen en lugares muy específicos. }\end{array}$ & $\begin{array}{l}\text { Investigar debe ser nuestra tarea diaria, tenemos todos los insumos } \\
\text { para hacerlo y a través de esa investigación mejorar la calidad de la } \\
\text { educación. Ahora veo mi aula de clase como el espacio de construc- } \\
\text { ción de nuevos saberes, como la posibilidad de responder nuevas } \\
\text { preguntas y como el espacio de mejoramiento permanente. }\end{array}$ \\
\hline
\end{tabular}

Fuente: elaboración propia (mayo de 2015)

5. Esta rutina está en el grupo de las rutinas que sintetizan y organizan ideas. Los principales tipos de pensamiento involucrados son la reflexión y la metacognición. Se usa para ayudar a estudiantes a analizar cómo las ideas que tenían sobre una temática han ido cambiando a medida que reciben nuevos conocimientos sobre ese tema en particular. 


\section{REFERENCIAS}

Álvarez Méndez, J. M. (2011). Evaluar pra conocer, examinar para excluir. Madrid: Morata.

Domingo, J. (2008). El aprendizaje cooperativo. Cuadernos de trabajo social, 21, 231 246.

Feldman, D. (2010). La evaluación. En: D. Feldman. Aportes para el desarrollo curricular. Didactica General (pp. 59-71). Buenos Aires: Ministerio de Educación de la Nación.

Jaramillo, R., Escobedo, H. y Bermúdez, Á. (octubre-noviembre-diciembre de 2004). Enseñanza para la Comprensión. Educere, Trasvase, 27, 529-534.

López, A. (2013). La evaluación herramienta para el aprendizaje. Bogotá: Magisterio.

Malbergier, M. y Campelo, A. (2009). La evaluación formativa. Escuela Primaria. Buenos Aires: Ministerio de Educación.
Martínez, J. A. (2007). La Enseñanza para la Comprensión: una aplicación en el aula. Bogotá: Universidad Pedagógica Nacional.

Mesa, L. G. (2009). Elementos de pensamiento crítico en Paulo Freire. Matemática, Educación e Internet, 10(1), 1-11.

Perkins, D. (1999). ¿Qué es la comprensión? En: M. Stone, La Enseñanza para la Comprensión (pp. 69-95). Buenos Aires: Paidós.

Perkins, D. (2003). ¿Cómo hacer visible el pensamiento?. Recuperado de: http://recursoseinnovacioneducativa.blogspot.com/2013/03/david-perkins.html

Ritchhart, R., Church, M., \& Morrison, K. (2011). Making Thinking Visible. San Francisco: Jossey-Bass.

Stone, M. (1999). ¿Qué es la Enseñanza para la Comprensión? En: M. Stone. La Enseñanza para la comprension. Vinculacion entre la investigacion y la práctica. (pp. 95-96). Buenos Aires: Paidós. 